\title{
D-galacturonic acid ameliorates the intestinal mucosal permeability and inflammation of functional dyspepsia in rats
}

\author{
Yu-Yao Wu ${ }^{1}$, Zi-Shao Zhong ${ }^{2}$, Zhen-Hao Ye ${ }^{2}$, Wang Zhang ${ }^{2}$, Gui-Hua $\mathrm{He}^{2}$, Yi-Feng Zheng ${ }^{1}$, \\ Sui-Ping Huang ${ }^{3}$
}

${ }^{1}$ Guangzhou University of Chinese Medicine, Guangzhou, China; ${ }^{2}$ The Second Clinical College of Guangzhou University of Chinese Medicine, Guangzhou, China; ${ }^{3}$ The Second Clinical College of Guangzhou University of Chinese Medicine, Guangdong Provincial Key Laboratory of Clinical Research on Traditional Chinese Medicine Syndrome, The Second Affiliated Hospital of Guangzhou University of Chinese Medicine, Guangzhou, China

Contributions: (I) Conception and design: ZS Zhong, W Zhang; (II) Administrative support: SP Huang, ZH Ye; (III) Provision of study materials or patients: YY Wu, YF Zheng; (IV) Collection and assembly of data: YY Wu, GH He; (V) Data analysis and interpretation: YY Wu; (VI) Manuscript writing: All authors; (VII) Final approval of manuscript: All authors.

Correspondence to: Professor Sui-Ping Huang. The Second Clinical College of Guangzhou University of Chinese Medicine, No.111, Dade Road, Yuexiu District, Guangzhou 510120, China. Email: gzdoctorhsp@126.com.

Backgrounda Functional dyspepsia (FD) is a gastrointestinal disease caused by imbalanced gastrointestinal
function. Traditional treatments are deemed to be limited, and new therapeutic drugs are required. New
study suggested that duodenal low-grade inflammation and increased intestinal permeability play an
important role in the pathogenesis of FD. Previous studies have shown that polysaccharides containing
D-galacturonic acid (GA) could modulate intestinal immune activity in vitro and in animal models. However,
the ability of GA monomer to improve intestinal mucosal permeability and inflammation in FD has not been
clearly elucidated.

Methods: A FD rat model was established using iodoacetamide (IA). FD Rats were administrated different doses of GA. Subsequently, the body weight and behavioral sensitivity of the rats were measured and evaluated; the permeability of the intestinal barrier was measured by determining D-lactose, lactulose/ mannitol ratio (LMR), and permeability-related genes [desmocollin-2 (DSC2), T7P1, and OCLN] in FD rats. Also, inflammatory cells [cluster of differentiation (CD)3+ cells and mast cells] were assessed by immunohistochemistry, and the levels of immune-related factors, such as the Toll-like receptor-nuclear factor kappa B (TLR/NF-кB) pathway, were monitored by reverse transcription quantitative polymerase chain reaction (RT-qPCR) or western blot assays.

Results: Our results suggested that GA could markedly increase the body weight and attenuate the behavioral sensitivity of FD rats. Moreover, GA also has an obvious ameliorating effect on the intestinal mucosal permeability and inflammatory response of FD rats. Furthermore, we found that GA could markedly downregulate TLR2, TLR4, and NF-кB in FD rats.

Conclusions: These findings indicate that GA could significantly attenuate the intestinal mucosal permeability and inflammation FD rats. The effect of GA was partially mediated by the TLR/NF- $\kappa \mathrm{B}$ signaling pathway.

Keywords: D-galacturonic acid (GA); intestinal mucosal permeability; inflammation; functional dyspepsia (FD); Toll-like receptor (TLR); nuclear factor kappa B (NF- $\mathrm{B})$

Submitted Nov 16, 2020. Accepted for publication Dec 29, 2020.

doi: 10.21037/apm-20-2420

View this article at: http://dx.doi.org/10.21037/apm-20-2420 


\section{Introduction}

Functional dyspepsia (FD) is a disease of the gastrointestinal system, which is characterized persistent or recurrent symptoms, such as early satiety, upper abdominal pain, belching, regurgitation, heartburn, nausea, and vomiting, without organic structural causes (1). It has three subtypes based on the Rome IV criteria: epigastric pain syndrome (EPS), postprandial distress syndrome (PDS), and a third type that exhibits overlapping features of both $(2,3)$. Currently, the prevalence of FD is $40 \%$ in Western countries and $28 \%$ in Eastern countries (4), and the prevalence among uninvestigated dyspepsia is $20.8 \%$ (5). It has been reported that the pathogenesis of FD is multifactorial, including visceral hypersensitivity changes, gastrointestinal motility abnormalities, impaired gastric regulatory function, and nervous system dysfunction $(2,6)$. However, the etiology and pathogenesis of FD have not been fully clarified, the symptomatic treatment effect for FD is not completely satisfactory, and patients require repeated medical treatment. Therefore, further exploration of the pathogenesis of FD and therapeutic drugs is crucial.

Recently, it was found that the duodenum might be the pathogenic center of FD (7). There are two possible reasons for this: the elevated sensitivity of intestinal mucosa due to increased inflammation or permeability, and the changes of duodenal metabolites observed in FD individuals $(8,9)$. At present, the outcomes of traditional therapies for FD are limited, and include Helicobacter pylori eradication, acid suppressants, and prokinetic combinations $(10,11)$. Duodenal low-grade inflammation is a new pathogenesis, but traditional therapies are still selected by physicians, even new progress only focuses on gastric, nervus, or sensory perception. What's more, scarcely any new treatment relevant to duodenal low-grade inflammation were reported in literature. Therefore, therapeutic drugs targeting the duodenum may offer new therapeutic options. Since the integrity of the duodenal-mucosal barrier is significant to the maintenance of normal duodenal function, we speculated that duodenal inflammation or intestinal barrier dysfunction, such as high intestinal permeability, might be the causes of FD.

Codonopsis pilosula is a Chinese herb that has anti-oxidant and anti-inflammatory functions (12). D-galacturonic acid (GA) is a class of purified monomer extracted from the Codonopsis pilosula polysaccharide. Previous research has demonstrated that the polysaccharide from Codonopsis pilosula can modulate intestinal immune activity in vitro and in animal models $(13,14)$. GA, as the main component of the polysaccharide, has significant biological activities such as promoting the proliferation of bifidobacteria, preventing the adsorption of toxic substances, as well as anti-cancer functions (15-17). As a natural substance, it can also extract from pectin. In this study, we established a FD rat model using oral gavage iodoacetamide (IA). We assessed the changes in body weight and behavioral sensitivity of the FD rats, and evaluated the impacts of GA on the intestinal mucosal permeability and inflammation. Furthermore, we proposed a possible regulatory mechanism of GA in FD rats. Our results provide a promising basis for novel FD therapy, we also study the major anti-inflammatory components of Codonopsis pilosula in order to provide evidence for the target of compound therapy. We present the following article in accordance with the ARRIVE reporting checklist (available at http://dx.doi.org/10.21037/apm-20-2420).

\section{Methods}

\section{Animal}

According to previous research (18), 60 specific pathogen free (SPF)-grade healthy Sprague-Dawley (SD) rats (male) were purchased from the Medical Laboratory Animal Center of Guangdong. The procedure was authorized by the Ethics Committee of Laboratory Animals in the Academy of Guangdong Province Chinese Medicine, Guangdong, China (ethical approval number: 2017007). All protocols were designed in accordance with the National Institutes of Health Guide for the Care and Use of Laboratory animals (NIH Publications No. 8023, revised 1978). Rats were nurtured in a controlled environment: 20 $26^{\circ} \mathrm{C}$, humidity $40-70 \%$, ventilation rate $10-20$ per hour, $7^{\text {th }}$ air purity, and light controlled from 7:00 am to 7:00 pm.

\section{Establishment of the rat model}

GA reagent with a purity quotient of $97 \%$ was purchased from Solarbio Biotechnology, Beijing, China.

\section{Grouping}

The 60 rats were randomly divided into six groups (with 10 rats in each group): control group (no special handling), FD model group, FD model + GA (L) (administration of $200 \mathrm{mg} / \mathrm{kg}$ GA), FD model + GA (M) (administration of $400 \mathrm{mg} / \mathrm{kg}$ GA), FD model + GA (H) (administration of 
$800 \mathrm{mg} / \mathrm{kg} \mathrm{GA}$ ), and a positive control group [administration of pyrrolidine dithiocarbamate ammonium (PDTC), a nuclear factor kappa B (NF- $\kappa \mathrm{B})$ inhibitor]. We determined the use level according to the dosage of Codonopsis pilosula polysaccharide in the relative literature and validated by pre-experimental. There's no evidence suggested risk of high dose GA now and the administration of GA are safety at all three levels according to our pre-experiment. When the pups grew up to 10 days, all were pretreated intragastrically with $0.2 \mathrm{~mL} 0.1 \%$ IA in $2 \%$ sucrose once daily, which was sustained for 6 days (except for the control group, which received $0.2 \mathrm{~mL} 2 \%$ sucrose solution per day). Other methods to establish a rat model include drug injection (such as Clonidine injection, Atropine injection); drug administration (such as soybean oil administration), neonatal intervention (such as neonatal maternal separation), stress stimulation (such as restraint stress, tail clamping) and some other intervention like chronic highfat feeding. We choose IA method because this way is a classical and comprehensive.

\section{Treatment of the rat model}

After balanced feeding for 6 weeks (all rats had free access to water and a standard diet), treatment groups were orally fed with 200,400 , or $800 \mathrm{mg} / \mathrm{kg}$ GA daily for 14 days, the positive control group received intraperitoneal injection of $100 \mathrm{mg} / \mathrm{kg}$ PDTC (Selleck, USA) daily for 14 days, while the control group and the model group received $0.9 \%$ saline solution $(0.1 \mathrm{~mL} / \mathrm{kg})$. There are no adverse events during experiment. At the end of the procedure, the rats were euthanized by exsanguination, and tissue and blood samples were obtained for future experiments.

\section{Sensitivity testing and body weight comparison}

The degree of visceral sensitivity was documented with increased balloon distention. Rotundity balloons, which can expand to $2 \mathrm{~cm}$ in diameter, were prepared to test the sensitive behavior of 10 -week-old animals. After $12 \mathrm{~h}$ of fasting, five rats were taken from each group randomly and anesthetized. Balloons were placed into an incision along the greater curvature (fundus) of the rats' stomachs. A $5 \mathrm{~cm}$ catheter was connected to the balloon and went through the subcutaneous tissue, and was then fixed at the back of the rats' neck to avoid being gnawed. The sensitivity testing was performed after a 1-week recovery period. After 6 days, rats were placed in an interference less room and adapted for $2 \mathrm{~h}$. The catheter was connected to an injector and a piezometer. Each balloon was inflated to $10,20,40$, and $60 \mathrm{mmHg}$ pressure for 20 seconds at $10 \mathrm{~min}$ intervals. Behavioral responses were graded according to the abdominal withdrawal reflex test (19-21): Grade 0, no response to balloon expansion; Grade 1, slight head movement; Grade 2, squinting or drilling, transient dysphoria; Grade 3, crawling around without repose; and Grade 4, contraction of body muscles with whining. Two blinded observers recorded the rats' behavior independently at the same time.

\section{Immunobistochemical (IHC) staining}

Duodenal biopsy specimens were immersed in $4 \%$ polyformaldehyde with 50 times greater volume for $12 \mathrm{~h}$ at $4{ }^{\circ} \mathrm{C}$, and were subsequently dehydrated in a hydroextractor for $14 \mathrm{~h}$. After embedding, tissue samples were cut into $3.5 \mu \mathrm{m}$-thick sections using a rotary microtome (Leica, Germany). These sections were floated in a $42.5^{\circ} \mathrm{C}$ water bath, mounted onto adhesion microscope slides (Citoglas, China), and dried by airing. After deparaffinization and rehydration, the slides were disposed in accordance with the instructions of the Streptavidin-Biotin Complex kit (Bosterbio, China), and were incubated with anti-mast cell antibody (1:200, Abcam, UK) and anti-cluster of differentiation (CD) 3 antibody (1:100, Abcam, UK), at $4{ }^{\circ} \mathrm{C}$ for 12 h. Finally, Diaminobenzidine Staining Kit (DAB, Bosterbio, China) was chosen to complete IHC. The results of IHC were determined by ImageJ software (National Institutes of Health, USA).

\section{Enzyme-linked immunosorbent assay (ELISA)}

Abdominal aortic blood was collected with heparin lithium evacuated blood tubes. The blood was centrifuged at $14,000 \mathrm{~g}$ for $10 \mathrm{~min}$ at $4{ }^{\circ} \mathrm{C}$, and the plasma was transferred to new tubes. The level of D-lactic acid was quantified using a D-lactate Assay Kit (EDLC-100) (BioAssay Systems, USA) according to the manufacturer's instructions.

\section{Lactulose/mannitol assay}

The rats were orally administered $6 \%$ lactulose and $4 \%$ mannitol in $0.2 \mathrm{~mL}$ of water after fasting for $6 \mathrm{~h}$. After random selection, the rats were placed into sterilized metabolic cages without water deprivation. Urine was 
Table 1 The sequences of the primer

\begin{tabular}{lll}
\hline & Forward primer & Reverse primer \\
\hline TJP1 & CTTGACCAGTACCCACGA & TCAGAGGAGGAACAACTGC \\
OCLN & CTACTCCTCCAACGGCAA & AGTCATCCACGGACAAGG \\
DSC2 & CCTGGAATTGGCGTAGTT & TTGCGTTGAGGGATACAC \\
TLR-4 & GCCACCATTTACAGTTCGT & GTCTCAGGCAGGAAAGGA \\
TLR-2 & CCTGGAGGTGTTGGATGT & GCTTCTGGGAGTGCTTCA \\
NF- אB & CCAAAGACCCACCTCACC & CGCTTCTTCACACACTGGA \\
$\beta$-actin & AGTTGCGTTACACCCTTTC & CACCTTCACCGTTCCAGT \\
\hline
\end{tabular}

DSC2, desmocollin-2; TLR, Toll-like receptor; NF-кB, nuclear factor kappa B.

collected $24 \mathrm{~h}$ after oral administration. The level of urinary lactulose/mannitol was detected by high-performance liquid chromatography (HPLC).

\section{Reverse transcription quantitative polymerase chain reaction (RT-qPCR) assay}

The duodenum was quickly dissected and temporarily immersed in liquid nitrogen for rapid freezing. Total ribonucleic acid (RNA) was extracted from the duodenum $(50 \mathrm{mg})$ in each group using $1 \mathrm{~mL}$ ice-cold Trizol reagent (Thermo, USA). Complementary deoxyribonucleic acid (cDNA) was synthesized using the Reverse Transcription Reagent Kit (AG, China) according to the manufacturer's instructions. Gene expressions were determined using a $10 \mu \mathrm{L}$ volume of cDNA. The primer sequences are presented in Table 1.

\section{Western blot assay}

Radioimmunoprecipitation assay (RIPA) buffer (Biorad, USA) including protease inhibitor (Roche, Switzerland), was added to the duodenums of the rats in each group, and total proteins were isolated through centrifugation. After determining the concentration of tissue samples using the bicinchoninic acid (BCA) method, the proteins were separated by sodium dodecyl sulphate-polyacrylamide gel electrophoresis (SDS-PAGE) and transferred onto the polyvinylidene difluoride (PVDF) membrane (Millipore, USA). After blocking, the membranes were immersed in primary antibodies at $4{ }^{\circ} \mathrm{C}$ overnight, followed by a secondary antibody for $1 \mathrm{~h}$. Protein bands were detected using a chemiluminescence reagent (Thermo, USA).
The primary antibodies and sources were as follows: desmocollin-2 (DSC2) (1:1,000, Abcam, UK), $\beta$-catenin (1:1,000, Cell Signaling Technology, USA), Toll-like receptor 4 (TLR4, 1:1,000, Abcam, UK), NF-кB (1:2,000, Abcam, UK), and $\beta$-actin $(1: 3,000$, Cell Signaling Technology, USA).

\section{Statistical analysis}

The measurement data were presented as mean \pm standard deviation (SD). The experimental data were analyzed using SPSS 20.0 (International Business Machines Corporation, USA) software. The body weight and grade of sensitive behavior were analyzed by repeated measures. D-lactic acid, the lactulose/mannitol ratio (LMR), the expressions of genes and proteins, and the positive immunohistochemistry data were analyzed using one-way analysis of variance (ANOVA). The Legacy Dialogs analysis was used for nonnormal data, Kruskal-Wallis $\mathrm{H}$ analysis was used to compare intergroup data, and Mann-Whitney $U$ analysis was used to compare values between groups. $\mathrm{P}<0.05$ was regarded as statistically significant in all experiments.

\section{Results}

\section{$G A$ increased the body weight and reduced the behavioral sensitivity of FD model rats}

The data showed that the body weights of the FD model rats were reduced compared to the control rats, and were elevated following GA administration, especially in rats administrated with high doses of GA at 8,9 , and 10 weeks $(\mathrm{P}<0.05$, Figure $1 A)$. We also assessed the behavioral 
A

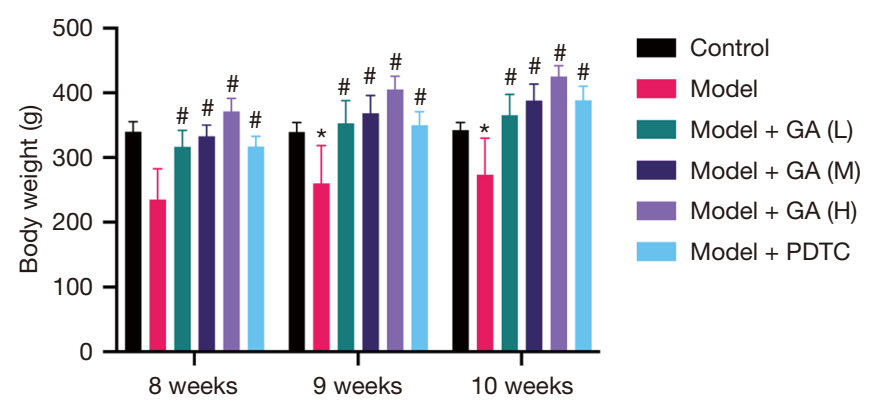

B

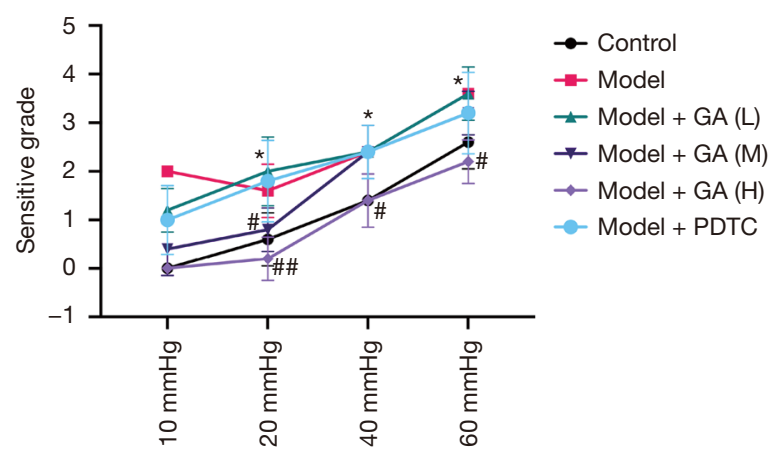

Figure 1 The expression of body weight gain and sensitive testing. (A) The body weights of each group at 8, 9, and 10 weeks(n=10). (B) The influence of GA on behavioral sensitivity $(\mathrm{n}=5)$. ${ }^{*} \mathrm{P}<0.05$ vs. the control group; ${ }^{\#} \mathrm{P}<0.05,{ }^{\# \#} \mathrm{P}<0.01$ vs. the model group. GA, galacturonic acid; PDTC, pyrrolidine dithiocarbamate ammonium.

sensitivity of rats in each group. As displayed in Figure $1 B$, behavioral sensitivity was increased in the FD model rats compared to the control rats, and GA administration attenuated the behavioral sensitivity of FD rats, especially high doses of $\mathrm{GA}(\mathrm{P}<0.05, \mathrm{P}<0.01)$. Overall, our data suggested that GA could promote increased body weight and weaken the behavioral sensitivity of FD rats.

\section{$G A$ reduced $C D 3+$ and mast cells in $F D$ rats}

As demonstrated in the IHC experiment, the proportion of $\mathrm{CD} 3$ + cells was increased in the FD model rats compared to the control rats, while CD3 + cells were lowered in GA-treated FD rats compared with the untreated groups $(\mathrm{P}<0.05, \mathrm{P}<0.01, \mathrm{P}<0.001$, Figure $2 A)$. Also, we found that the levels of mast cells were notably elevated in the FD model rats compared to the control rats. The administration of GA reduced the levels of mast cells, and the effect was dose dependent $(\mathrm{P}<0.05, \mathrm{P}<0.01, \mathrm{P}<0.001$, Figure $2 B)$. Thus, our data indicated that GA could significantly reduce inflammation in FD rats.

\section{$G A$ reduced the permeability of the intestinal barrier of FD model rats}

We observed that the level of D-lactose was prominently elevated in the FD model rats compared to the control rats, and that this elevation of $\mathrm{D}$-lactose could be reversed by increasing amounts of $\mathrm{GA}(\mathrm{P}<0.05, \mathrm{P}<0.01, \mathrm{P}<0.001$, Figure $3 A$ ). We also performed a lactulose/mannitol assay and found that the LMR was markedly enhanced in the FD model rats compared to the control rats. The administration of increasing amounts of GA suppressed the LMR $(\mathrm{P}<0.05$, $\mathrm{P}<0.01, \mathrm{P}<0.001$, Figure $3 B$ ). Overall, our findings suggested that GA could significantly ameliorate LMR and D-lactic acid levels in FD rats.

\section{GA up-regulated OCLN, THP1, DSC2 and $\beta$-catenin in FD rats}

As displayed in Figure 4A,B,C, OCLN, TfP1, and DSC2 were downregulated in the FD model rats relative to the control rats. In contrast, the administration of increasing amounts of GA reversed the expression of OCLN, TFP1, and DSC 2 in FD rats $(\mathrm{P}<0.05, \mathrm{P}<0.01, \mathrm{P}<0.001)$. Moreover, western blotting results confirmed that GA could upregulate DSC2 and $\beta$-catenin in $F D$ rats $(\mathrm{P}<0.05, \mathrm{P}<0.001$, Figure $4 D, E, F)$. Overall, our data confirmed that GA could modulate the intestinal mucosal permeability of FD rats.

\section{GA down-regulated TLR2, TLR4, and NF- $B$ B in FD rats}

We further explored the possible downstream genes that GA may regulate in FD rats. RT-qPCR analysis revealed that the messenger RNA (mRNA) levels of TLR2, TLR4, and NF- $\mathrm{KB}$ were notably upregulated in the FD model rats compared to the control rats, and that administration of GA decreased these mRNA levels $(\mathrm{P}<0.05, \mathrm{P}<0.01, \mathrm{P}<0.001$, Figure $5 A, B, C)$. Similarly, Western blot data confirmed that GA could reduce the expression of TLR4 and NF- $\kappa B$ in FD rats, and the regulation effect of GA at a high dose was more obvious than at a low dose $(\mathrm{P}<0.05, \mathrm{P}<0.01, \mathrm{P}<0.001$, 
A
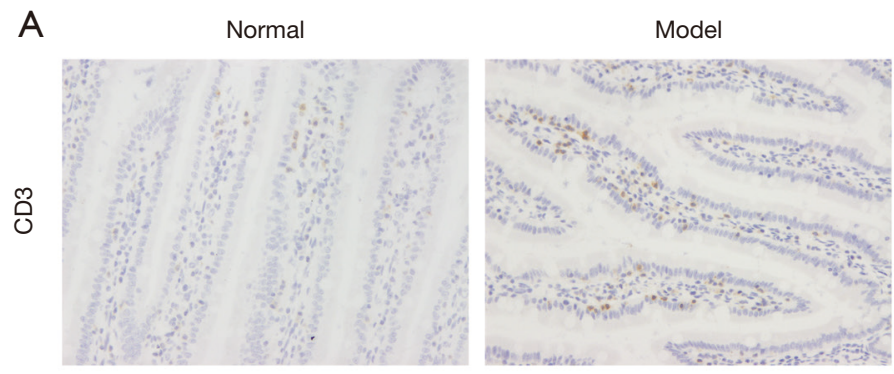

B

Normal

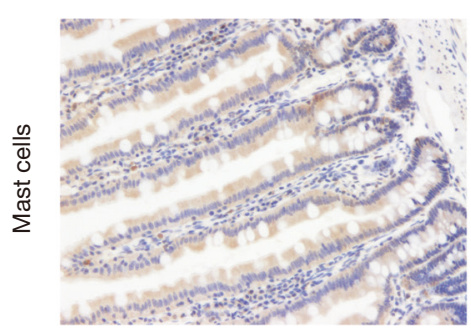

Model

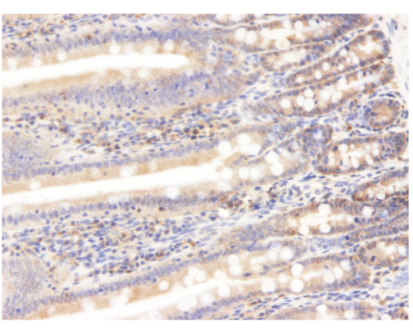

Model + GA

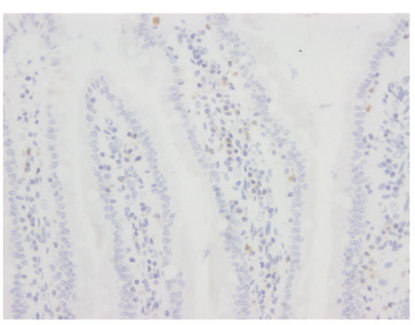

Model + GA

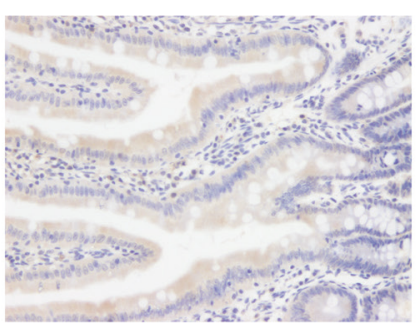

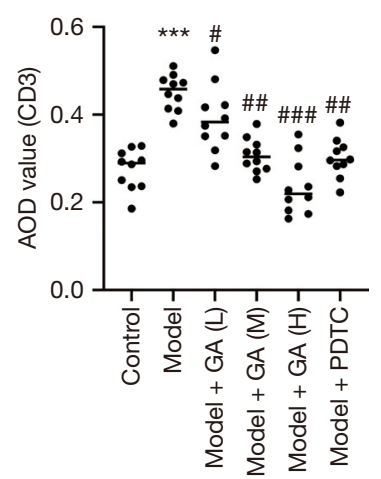

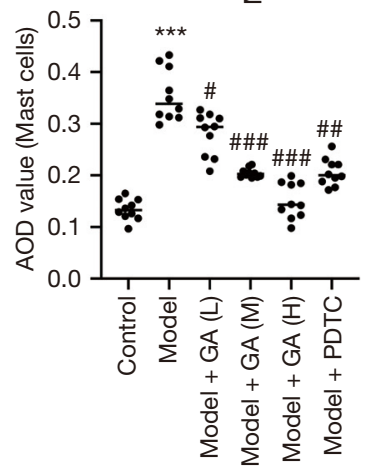

Figure 2 The expression changes of (A) CD3+ cells and (B) mast cells in FD rats. The quantitative analysis was performed in accordance with the average optical density (AOD) value. The staining method of (A) and (B) was diaminobenzidine staining. Original magnification: $\times 20$. $(\mathrm{n}=10)$. ${ }^{* * *} \mathrm{P}<0.001$ vs. the control group; ${ }^{\#} \mathrm{P}<0.05,{ }^{\# \#} \mathrm{P}<0.01,{ }^{\# \#} \mathrm{P}<0.001$ vs. the model group. GA, galacturonic acid; $\mathrm{PDTC}$, pyrrolidine dithiocarbamate ammonium; FD, functional dyspepsia; AOD, average optical density.
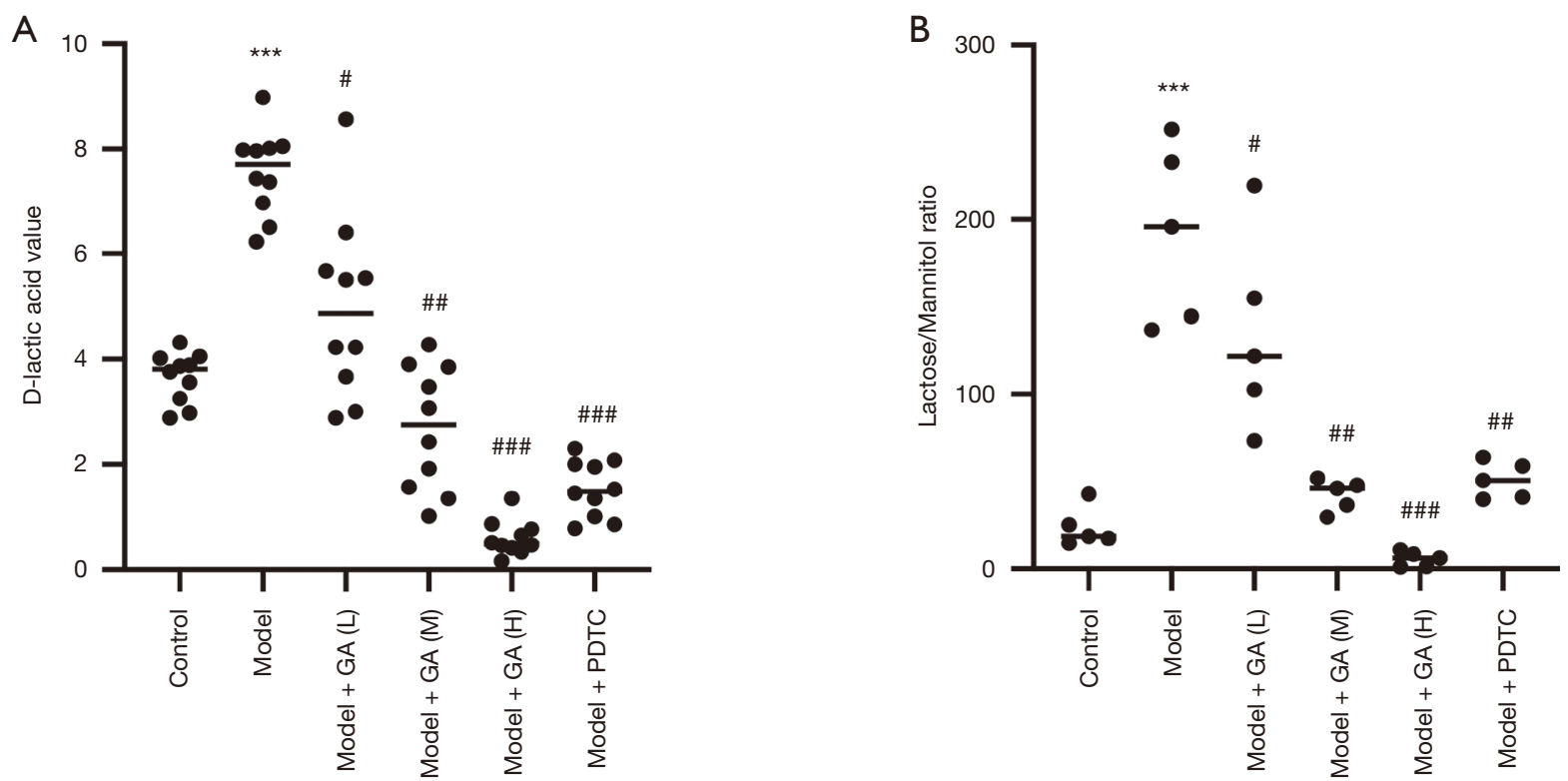

Figure 3 The expression of D-lactic acid and lactulose/mannitol ratio(n=10). (A) The level of D-lactose in the rats of each group. (B) The degree of $\mathrm{LMR}$ in rats of each group. ${ }^{* * *} \mathrm{P}<0.001$ vs. control group; ${ }^{\#} \mathrm{P}<0.05,{ }^{\# \#} \mathrm{P}<0.01,{ }^{\# \# \#} \mathrm{P}<0.001$ vs. model group. GA, galacturonic acid; PDTC, pyrrolidine dithiocarbamate ammonium; LMR, lactulose/mannitol ratio. 
A

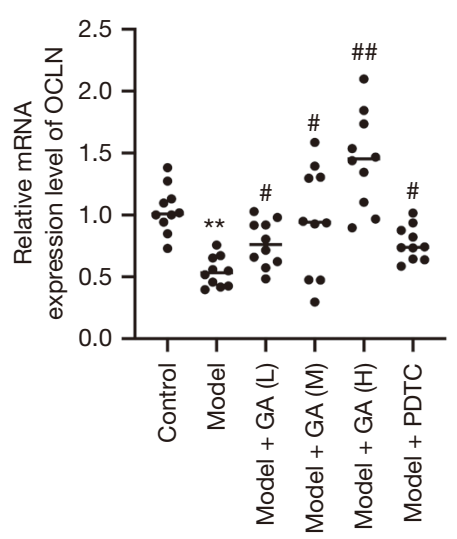

$\mathrm{D}$

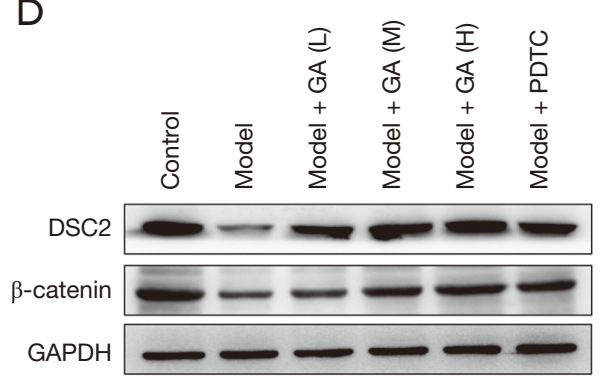

B

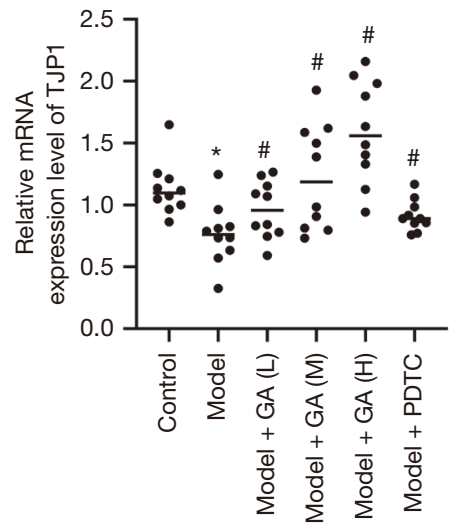

E

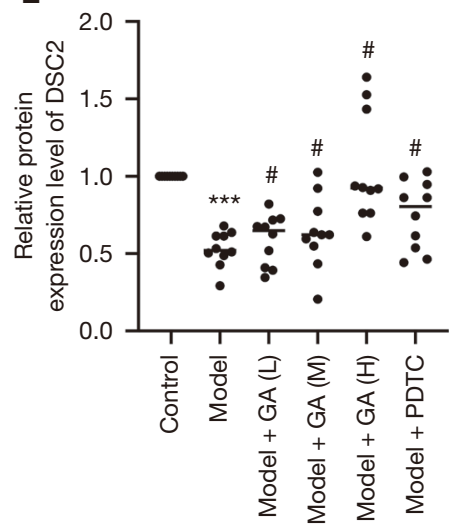

C

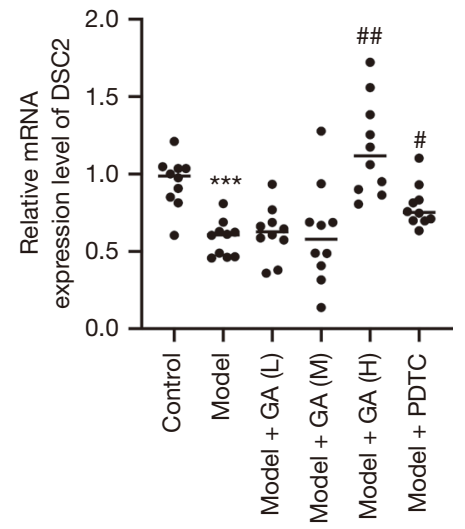

$\mathrm{F}$

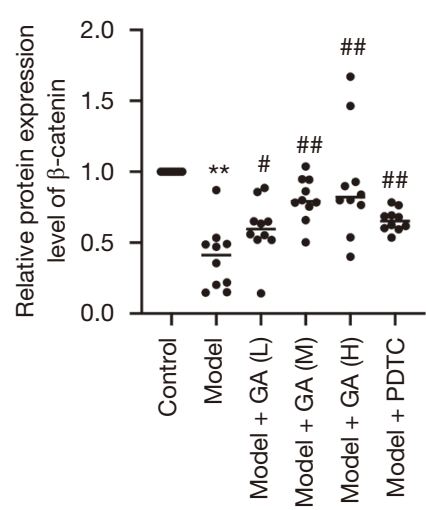

Figure 4 The expression of factors related to duodenum mucosal permeability (n=10). GA upregulated OCLN, TFP1, DSC2, and $\beta$-catenin in FD rats. (A,B,C) RT-qPCR analysis was applied to confirm the mRNA expression levels of OCLN, TFP1, and DSC2. (D) DSC2 and $\beta$-catenin expression was evaluated using Western blotting. Quantitative analysis of (E) DSC2 and (F) $\beta$-catenin proteins was conducted based on the grayscale values. ${ }^{*} \mathrm{P}<0.05,{ }^{* *} \mathrm{P}<0.01,{ }^{* * *} \mathrm{P}<0.001$ vs. control group; ${ }^{\#} \mathrm{P}<0.05,{ }^{\# \#} \mathrm{P}<0.01$ vs. model group. GA, galacturonic acid; PDTC, pyrrolidine dithiocarbamate ammonium; FD, functional dyspepsia; DSC2, desmocollin-2; RT-qPCR, reverse transcription quantitative polymerase chain reaction.

Figure $5 D, E, F)$. In summary, our data suggested that the TLR/NF- $\kappa B$ pathway could be suppressed by GA in FD rats, suggesting that the TLR/NF- $\mathrm{KB}$ pathway is likely involved in the remission effect of GA in FD rats.

\section{Discussion}

Our study demonstrated that the FD rats had slight inflammation and high intestinal permeability in the duodenum, and that the intestines might be a new onset position of FD instead of the stomach. GA had a significant reduction effect on inflammation and intestinal mucosal permeability in the duodenum of FD rats. Additionally, we found GA could dramatically downregulate TLR/NF- $\mathrm{KB}$ signaling in FD rats, which indicated that the TLR/NF$\kappa \mathrm{B}$ signaling pathway might be related to the pathogenesis of FD.

In accordance with the least-significant difference (LSD) test, the body weight of rats was remarkably increased after treatment with GA or PDTC. Significant differences appeared both between groups and between weeks, which demonstrated that a higher value of GA and a longer treatment period led to a better outcome. According to a previous study (18), IA can strip the gastrointestinal mucous coating of rats, which could lead to transient gastritis during the neonatal period, especially from day 3 to day 6 . Our study suggested that this influence could extend to adulthood, and that a high dosage of GA can result in the 
A

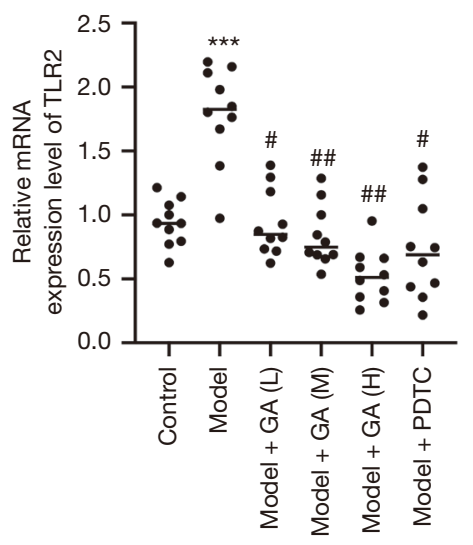

$\mathrm{D}$

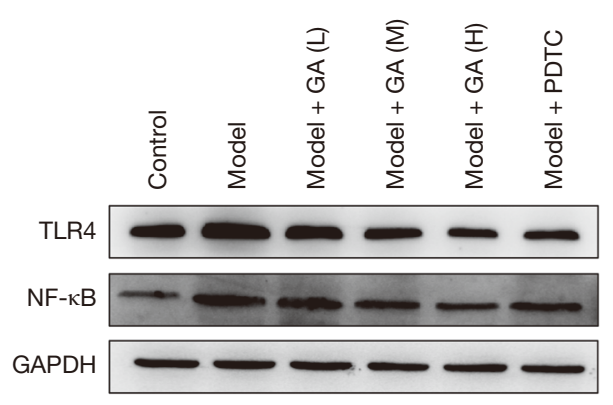

B

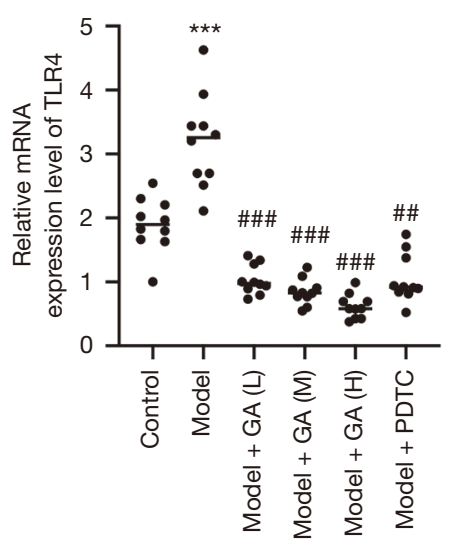

$\mathrm{E}$

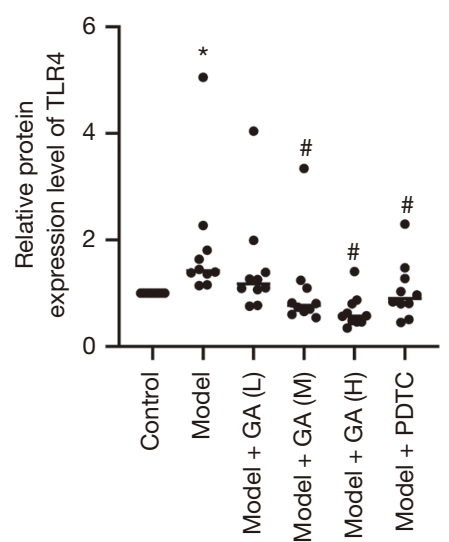

C

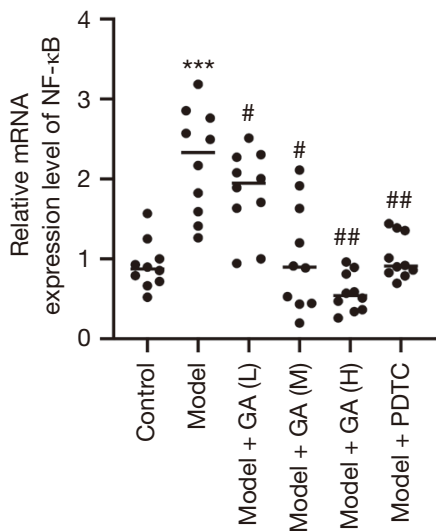

$\mathrm{F}$

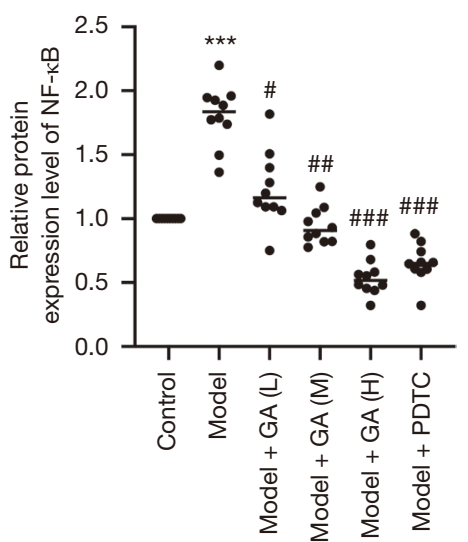

Figure 5 The expression of factors related to duodenum inflammation $(\mathrm{n}=10)$. (A,B,C) GA down-regulated TLR2, TLR4, and NF-kB in FD rats. (D) TLR2, TLR4, and NF-kB expressions were confirmed using RT-qPCR analysis. (E,F) Western blotting analysis was conducted to

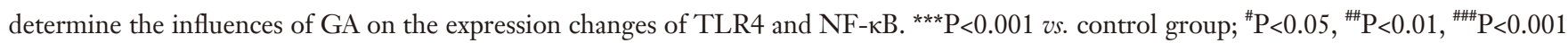
vs. model group. GA, galacturonic acid; PDTC, pyrrolidine dithiocarbamate ammonium; FD, functional dyspepsia; RT-qPCR, reverse transcription quantitative polymerase chain reaction; TLR, Toll-like receptor; NF-kB, nuclear factor kappa B.

most obvious recovery. Our results also indicated that GA could markedly reduce the behavioral sensitivity of FD model rats, since there was little difference between the high dose group and the control group, suggesting that GA has a significant therapeutic effect on the behavioral sensitivity of FD rats.

There is a consensus in the literature that patients with functional gastrointestinal disorder have increased levels of mucosal immune cells (22), and some studies have reported that the occurrence of FD can increase the amount of immune cells in the duodenum (23-25). In our study, we demonstrated that the levels of CD3 + cells and mast cells were notably increased in FD rats, suggesting that FD could trigger immune activation in duodenal tissues, which may further lead to abdominal pain and other gastrointestinal disorders. We also demonstrated that GA could reduce inflammatory cells in the duodenum of FD rats, suggesting that GA could attenuate inflammation in FD rats.

Generally, D-lactic acid is seldom present in the intestinal lumen, and its concentration could signal initial damage to the intestinal barrier. In our study, we found that GA could significantly reduce the levels of D-lactose, a reliable biomarker of intestinal permeability (26). Similarly, since only a small proportion of lactulose could permeate through the intestinal mucosa, while mannitol could always pass through, intestinal permeability can be measured by detecting the LMR in the urine (27). We found that treatment with GA can restore the LMR to the 
control level. The causes of intestinal barrier dysfunction are multifactorial, and include increased intestinal mucosal permeability, impaired tight junctions of the epithelial barrier, destruction of intestinal flora, and invasive harmful metabolites. As revealed by endoscopy, the infiltration of immune cells was found to arise simultaneously with increased paracellular permeability and decreased epithelial resistance in biopsy samples (28). One study discovered that the adherent junctions exhibited larger spaces in FD biopsy samples (29). Moreover, a recent study suggested that there is a correlation between impaired duodenal mucosal integrity characterized by low inflammation in the process of FD (30). Since the intestinal barrier plays a key role in preventing noxious contents, increased intestinal permeability would contribute to intrusive stimuli, such as dietary antigens, across the intestinal mucosa, which both lead to systemic inflammatory responses. Therefore, we suggested that GA could not only modulate immune activation, but also has a beneficial effect on the mucosal barrier.

$\mathrm{NF}-\mathrm{\kappa B}$ is a multifunctional nuclear transcription factor in eukaryotic cells, which is involved in both the immune and inflammatory responses $(31,32)$. The TLR/NF- $\kappa B$ pathway is associated with the pathogenic factors of $\mathrm{FD}$, such as gastrointestinal microecological abnormalities, stress, infection, and dietary antigens. Abnormal activation of the TLR/NF- $\kappa B$ pathway can result in a sustained immune response and inflammatory damage in the gastrointestinal tract (33). The results of our study demonstrated that GA could notably downregulate TLR2, TLR4, and NF$\kappa \mathrm{B}$ in FD rats at both the mRNA and the protein levels, indicating the crucial role of the TLR/NF- $\kappa \mathrm{B}$ pathway in the remission effect of GA in FD rats. However, more detailed study is needed to verify whether the TLR/NF$\kappa \mathrm{B}$ pathway can be targeted in the treatment of FD in the future. The findings would translate to human biology after the mechanism study completed.

\section{Acknowledgments}

Funding: This study was funded by the Science and Technology Planning Project of Guangdong Province (grant numbers 2017B030314166 and YN2018ZD09), and the Traditional Chinese Medicine Bureau of Guangdong province (grant number 2019KT1045), which are used for purchasing experimental rats, reagents, laboratory consumptive materials.

\section{Footnote}

Reporting Checklist: The authors have completed the ARRIVE reporting checklist. Available at http://dx.doi. org/10.21037/apm-20-2420

Data Sharing Statement: Available at http://dx.doi. org/10.21037/apm-20-2420

Conflicts of Interest: All authors have completed the ICMJE uniform disclosure form (available at http://dx.doi. org/10.21037/apm-20-2420). The authors have no conflicts of interest to declare.

Ethical Statement: The authors are accountable for all aspects of the work in ensuring that questions related to the accuracy or integrity of any part of the work are appropriately investigated and resolved. The procedure was authorized by the Ethics Committee of Laboratory Animals in the Academy of Guangdong Province Chinese Medicine, Guangdong, China (ethical approval number: 2017007). All protocols were designed in accordance with the National Institutes of Health Guide for the Care and Use of Laboratory animals (NIH Publications No. 8023, revised 1978).

Open Access Statement: This is an Open Access article distributed in accordance with the Creative Commons Attribution-NonCommercial-NoDerivs 4.0 International License (CC BY-NC-ND 4.0), which permits the noncommercial replication and distribution of the article with the strict proviso that no changes or edits are made and the original work is properly cited (including links to both the formal publication through the relevant DOI and the license). See: https://creativecommons.org/licenses/by-nc-nd/4.0/.

\section{References}

1. Enck P, Azpiroz F, Boeckxstaens G, et al. Functional dyspepsia. Nat Rev Dis Primers 2017;3:17081.

2. Talley NJ, Ford AC. Functional Dyspepsia. N Engl J Med 2015;373:1853-63.

3. Walker MM, Talley NJ. Functional Dyspepsia in the Elderly. Curr Gastroenterol Rep 2019;21:54.

4. Mahadeva S, Ford AC. Clinical and epidemiological differences in functional dyspepsia between the East and the West. Neurogastroenterol Motil 2016;28:167-74.

5. Ford AC, Marwaha A, Sood R, et al. Global prevalence 
of, and risk factors for, uninvestigated dyspepsia: a metaanalysis. Gut 2015;64:1049-57.

6. Tomita T, Oshima T, Miwa H. New Approaches to Diagnosis and Treatment of Functional Dyspepsia. Curr Gastroenterol Rep 2018;20:55.

7. Miwa H, Oshima T, Tomita T, et al. Recent understanding of the pathophysiology of functional dyspepsia: role of the duodenum as the pathogenic center. J Gastroenterol 2019;54:305-11.

8. Jung HK, Talley NJ. Role of the Duodenum in the Pathogenesis of Functional Dyspepsia: A Paradigm Shift. J Neurogastroenterol Motil 2018;24:345-54.

9. Wauters L, Talley NJ, Walker MM, et al. Novel concepts in the pathophysiology and treatment of functional dyspepsia. Gut 2020;69:591-600.

10. Oshima T, Miwa H. Functional Dyspepsia - A Revolution in Management. Am J Gastroenterol 2018;113:1420-2.

11. Talley NJ. Functional dyspepsia: new insights into pathogenesis and therapy. Korean J Intern Med 2016;31:444-56.

12. Jiang Y, Liu Y, Guo Q, et al. Acetylenes and fatty acids from Codonopsis pilosula. Acta Pharmaceutica Sinica B 2015;5:215-22.

13. Zou YF, Zhang YY, Fu YP, et al. A Polysaccharide Isolated from Codonopsis pilosula with Immunomodulation Effects Both In Vitro and In Vivo. Molecules 2019;24:3632.

14. Zheng YS, Wu ZS, Ni HB, et al. Codonopsis pilosula polysaccharide attenuates cecal ligation and puncture sepsis via circuiting regulatory $\mathrm{T}$ cells in mice. Shock 2014;41:250-5.

15. Protzko RJ, Hach CA, Coradetti ST, et al. Genomewide and Enzymatic Analysis Reveals Efficient d-Galacturonic Acid Metabolism in the Basidiomycete Yeast Rhodosporidium toruloides. mSystems 2019;4:e00389-19.

16. Jimenez AG, Ellermann $M$, Abbott $W$, et al. Diet-derived galacturonic acid regulates virulence and intestinal colonization in enterohaemorrhagic Escherichia coli and Citrobacter rodentium. Nat Microbiol 2020;5:368-78.

17. Martín-Rodríguez F, López-Izquierdo R, Castro Villamor MA, et al. Prognostic value of lactate in prehospital care as a predictor of early mortality. Am J Emerg Med 2019;37:1627-32.

18. Liu LS, Winston JH, Shenoy MM, et al. A rat model of chronic gastric sensorimotor dysfunction resulting from transient neonatal gastric irritation. Gastroenterology 2008;134:2070-9.

19. Tang QL, Lai ML, Zhong YF, et al. Antinociceptive effect of berberine on visceral hypersensitivity in rats. World $\mathrm{J}$
Gastroenterol 2013;19:4582-9.

20. Yang JP, Yao M, Jiang XH, et al. Establishment of model of visceral pain due to colorectal distension and its behavioral assessment in rats. World J Gastroenterol 2006;12:2781-4.

21. Wang $\mathrm{Y}, \mathrm{Qu} \mathrm{R}, \mathrm{Hu} \mathrm{S}$, et al. Upregulation of cystathionine beta-synthetase expression contributes to visceral hyperalgesia induced by heterotypic intermittent stress in rats. PLoS One 2012;7:e53165.

22. Spiller RC, Jenkins D, Thornley JP, et al. Increased rectal mucosal enteroendocrine cells, T lymphocytes, and increased gut permeability following acute Campylobacter enteritis and in post-dysenteric irritable bowel syndrome. Gut 2000;47:804-11.

23. Du L, Chen B, Kim JJ, et al. Micro-inflammation in functional dyspepsia: A systematic review and metaanalysis. Neurogastroenterol Motil 2018;30:e13304.

24. Talley NJ, Holtmann G, Walker MM. Therapeutic strategies for functional dyspepsia and irritable bowel syndrome based on pathophysiology. J Gastroenterol 2015;50:601-13.

25. Liebregts T, Adam B, Bredack C, et al. Small bowel homing $\mathrm{T}$ cells are associated with symptoms and delayed gastric emptying in functional dyspepsia. Am J Gastroenterol 2011;106:1089-98.

26. Kong C, Li SM, Yang H, et al. Screening and combining serum biomarkers to improve their diagnostic performance in the detection of intestinal barrier dysfunction in patients after major abdominal surgery. Ann Transl Med 2019;7:388.

27. Warners MJ, Vlieg-Boerstra BJ, Verheij J, et al. Esophageal and Small Intestinal Mucosal Integrity in Eosinophilic Esophagitis and Response to an Elemental Diet. Am J Gastroenterol 2017;112:1061-71.

28. Ishigami H, Matsumura T, Kasamatsu S, et al. EndoscopyGuided Evaluation of Duodenal Mucosal Permeability in Functional Dyspepsia. Clin Transl Gastroenterol 2017;8:e83.

29. Tanaka F, Tominaga K, Fujikawa Y, et al. Concentration of Glial Cell Line-Derived Neurotrophic Factor Positively Correlates with Symptoms in Functional Dyspepsia. Dig Dis Sci 2016;61:3478-85.

30. Potter MDE, Walker MM, Jones MP, et al. Wheat Intolerance and Chronic Gastrointestinal Symptoms in an Australian Population-based Study: Association Between Wheat Sensitivity, Celiac Disease and Functional Gastrointestinal Disorders. Am J Gastroenterol 2018;113:1036-44.

31. Cildir G, Low KC, Tergaonkar V. Noncanonical NF- 
$\kappa \mathrm{B}$ Signaling in Health and Disease. Trends Mol Med 2016;22:414-29.

32. Fan Y, Mao R, Yang J. NF-кB and STAT3 signaling pathways collaboratively link inflammation to cancer. Protein Cell 2013;4:176-85.

33. Castaño-Rodríguez N, Kaakoush NO, Goh KL, et al. The role of TLR2, TLR4 and CD14 genetic polymorphisms in gastric carcinogenesis: a case-control study and metaanalysis. PLoS One 2013;8:e60327.

(English Language Editor: A. Kassem)

Cite this article as: $\mathrm{Wu} Y Y$, Zhong ZS, Ye ZH, Zhang W, He GH, Zheng YF, Huang SP. D-galacturonic acid ameliorates the intestinal mucosal permeability and inflammation of functional dyspepsia in rats. Ann Palliat Med 2021;10(1):538-548. doi: 10.21037/apm-20-2420 